\title{
Numerical simulation for fractional-order differential system of a Glioblastoma Multiforme and Immune system
}

\author{
M.M. Al-Shomrani ${ }^{1}$ and M.A. Abdelkawy ${ }^{2,3^{*}}$ (D)
}

\author{
"Correspondence: \\ melkawy@yahoo.com \\ ${ }^{2}$ Department of Mathematics, \\ Faculty of Science, Beni-Suef \\ University, Beni-Suef, Egypt \\ ${ }^{3}$ Department of Mathematics and \\ Statistics, College of Science, Imam \\ Mohammad Ibn Saud Islamic \\ University (IMSIU), Riyadh, Saudi \\ Arabia \\ Full list of author information is \\ available at the end of the article
}

\begin{abstract}
In this paper, we present a numerical simulation to study a fractional-order differential system of a glioblastoma multiforme and immune system. This numerical simulation is based on spectral collocation method for tackling the fractional-order differential system of a glioblastoma multiforme and immune system. We introduce new shifted fractional-order Legendre orthogonal functions outputted by Legendre polynomials. Also, we state and derive some corollaries and theorems related to the new shifted fractional order Legendre orthogonal functions. The shifted fractional-order Legendre-Gauss-Radau collocation method is developed to approximate the fractional-order differential system of a glioblastoma multiforme and immune system. The basis of the shifted fractional-order Legendre orthogonal functions is adapted for temporal discretization. The solution of such an equation is approximated as a truncated series of shifted fractional-order Legendre orthogonal functions for temporal variable, and then we evaluate the residuals of the mentioned problem at the shifted fractionalorder Legendre-Gauss-Radau quadrature points. The accuracy of the novel method is demonstrated with several test problems.
\end{abstract}

MSC: 65M70; 41A55; 26A33

Keywords: Spectral collocation method; Gauss-Radau quadrature; Shifted Legendre polynomials; Caputo fractional derivative; Glioblastoma multiforme; Immune system

\section{Introduction}

Glioblastoma, also known as glioblastoma multiforme (GBM), is the most deadly cancer that infects the brain. Cancer is one of the vicious killers in the world and the control of tumor growth requires special attention [1]. The typical approach for treating GBM involves surgical resection of as much of the tumor as possible, followed by radiation treatment and chemotherapy [2]. Within a single tumor of monoclonal origin, this kind of tumor can develop multiple sub-populations, each of which may be characterized by different growth rates and treatment susceptibilities [3-5]. Works about modeling of multi sub-populations can be shown in $[1,6]$. Modeling the interaction between the tumor cell and the immune system is interesting research, for a few of research works, see $[1,7,8]$.

(c) The Author(s) 2020. This article is licensed under a Creative Commons Attribution 4.0 International License, which permits use sharing, adaptation, distribution and reproduction in any medium or format, as long as you give appropriate credit to the original author(s) and the source, provide a link to the Creative Commons licence, and indicate if changes were made. The images or other third party material in this article are included in the article's Creative Commons licence, unless indicated otherwise in a credit line to the material. If material is not included in the article's Creative Commons licence and your intended use is not permitted by statutory regulation or exceeds the permitted use, you will need to obtain permission directly from the copyright holder. To view a copy of this licence, visit http://creativecommons.org/licenses/by/4.0/. 
A large amount of work on modeling biological systems has been restricted to fractionalorder ordinary differential equations [9-12]. In the last decades, fractional calculus theory [13] has been developed rapidly. It has been applied in several scientific areas such as engineering, viscoelasticity, physics, diffusion processes, rheology, etc. [14-17]. Actually, fractional calculus theory is considered as a substitution model to classical calculus theory. Fractional differential equations including those of fixed, variable, distributed, tempered order have been used as perfect models for real world problems including earthquake analysis, viscoelasticity, bio-chemical, controller design, electric circuits, diffusion processes, signal processing, etc. [18-20]. Therefore, the urgent necessity to find the exact solutions or merely the approximate ones to these problems has emerged. Since the finding of the exact solutions is not possible for most fractional differential equations [21]; the numerical methods have been developed to obtain the approximate solutions to them.

Some local numerical techniques have been introduced for solving fractional-order biological systems $[22,23]$, and this method may become computationally heavy due to the nonlocal property of fractional differential operator. Moreover, the local methods listed the approximate solution at specific points, while the global methods give the approximate solution in the whole mentioned interval. Hence, the global behavior of the solution can be naturally taken into account. In many areas of science such as engineering, biology, economics, physics, and others, several high-order numerical methods have been developed to deal with the related problems. Among these algoriths, spectral methods [24, 25] have been rapidly developed in the last four decades. Spectral methods [26, 27] are widely applied as powerful techniques in the construction of numerical solution for differential and fractional differential equations.

The spectral collocation method, a global numerical technique [28-30], is a particular kind of famous spectral methods that is widely applicable for almost all types of differential equations. The convergence speed is one of the major advantages of the spectral method. Spectral methods have exponential convergence rates as well as high accuracy level. Thus, the spectral collocation method is a more reliable, suitable, and accurate technique for treating such problems. In many areas of science, such as engineering, biology, economics, physics, and others, several high-order numerical methods have been developed to deal with the related problems. Recently, spectral methods have been known as efficient and highly accurate schemes. The spectral method is classified into four kinds, namely collocation [28-30], Tau [31], Galerkin [32], and Petrov-Galerkin [33] methods.

Here, a shifted fractional-order Legendre-Gauss-Radau collocation (SFL-GR-C) method is developed to approximate the fractional-order differential system of a glioblastoma multiforme and immune system (GBM-IS). In this paper, we want to numerically solve the fractional-order differential system of GBM-IS [34-36]

$$
\begin{aligned}
& D^{\mu} S(t)=p S(t)+r_{1} S(t)\left(k_{1}-\beta_{1} S(t)\right)-\gamma S(t) R(t)-d_{1} S(t)-\tau_{1} S(t) N(t), \\
& S(0)=S_{0}, \\
& D^{\mu} R(t)=r_{2} R(t)\left(k_{2}-\beta_{2} R(t)\right)+\gamma S(t) R(t)-d_{2} R(t)-\tau_{2} R(t) N(t), \\
& R(0)=R_{0}, \\
& D^{\mu} N(t)=\delta N(t) Z(t)-\epsilon Z(t) N(t)-d_{3} N(t), \quad N(0)=N_{0},
\end{aligned}
$$




$$
\begin{aligned}
& D^{\mu} Z(t)=r_{3} Z(t)\left(k_{3}-\beta_{3} Z(t)\right)-\delta N(t) Z(t)+\epsilon Z(t) N(t)-d_{4} Z(t), \\
& Z(0)=Z_{0} .
\end{aligned}
$$

Here, the temporal derivative is taken in the Caputo sense with fractional order $\mu$, $0<\mu<1$. Where the parameters $\beta_{1}, \beta_{2}, \beta_{3}, \tau_{1}, \tau_{2}, \gamma, \delta, \epsilon, p, d_{1}, d_{2}, d_{3}, d_{4}, k_{1}, k_{2}, k_{3}$, $r_{1}, r_{2}$, and $r_{3}$ are positive constants. $S(t), R(t), N(t)$, and $Z(t)$ are used for sensitive tumor cell, resistant tumor cell, activated macrophages, and macrophages, respectively, where $p$ equals division rate of the sensitive tumor cell, $k_{1}$ and $k_{2}$ are the carrying capacities of the sensitive (including necrotic part) and resistant cell, respectively. Parameters $\beta_{1}$ and $\beta_{2}$ are necessary to construct logistic differential equations. $r_{1}$ and $r_{2}$ are the growth rate of the sensitive cell and the resistant cell, respectively. $\gamma$ is the converting rate of the sensitive tumor cell to the resistant tumor cell. Parameters $d_{1}$ and $d_{2}$ are their dead rate caused from drugs, respectively. $\delta$ is the conversion rate of macrophages to active macrophages. $d_{3}$ is the natural death of active macrophages and $d_{4}$ is the natural death of macrophages. $r_{3}$ is the growth rate of macrophages and the parameter $\beta_{3}$ is selected in view of logistic differential equations. $k_{3}$ gives the carrying capacity of macrophages. $\epsilon$ is the conversion rate of the activated macrophages to macrophages. $\tau_{1}$ and $\tau_{2}$ are the destroying rate caused by the activated macrophages.

The rest of this paper is organized as follows. In the next section, we introduce several analytical and numerical prerequisites. In Sect. 3, we construct a spectral numerical scheme. In Sect. 4, three numerical examples are presented to support our theoretical results and to demonstrate the significant gain in accuracy. Finally, we give a brief conclusion in Sect. 5.

\section{Legendre polynomials}

We recall that the Legendre polynomials $\Omega_{k}(t)(k=0,1,2, \ldots)$ obey the Rodrigues formula, namely

$$
\Omega_{k}(t)=\frac{(-1)^{k}}{2^{k} k !} D^{k}\left(\left(1-t^{2}\right)^{k}\right)
$$

Accordingly, $\Omega_{k}^{(p)}(t)$ (the $p$ th derivative of $\left.\Omega_{k}(t)\right)$ is given by

$$
\Omega_{k}^{(p)}(t)=\sum_{i=0(\tau+k=\text { even })}^{k-p} C_{p}(k, \tau) P_{\tau}(t)
$$

where

$$
C_{p}(k, \tau)=\frac{2^{p-1}(2 \tau+1) \Gamma\left(\frac{p+k-\tau}{2}\right) \Gamma\left(\frac{p+k+\tau+1}{2}\right)}{\Gamma(p) \Gamma\left(\frac{2-p+k-\tau}{2}\right) \Gamma\left(\frac{3-p+k+\tau}{2}\right)} .
$$

Next, denote by $\chi$ and $(\chi, v)$ the norm and the inner product of space $L^{2}[-1,1]$.

The set of $\Omega_{k}(t)$ is a complete orthogonal system in $L^{2}[-1,1]$

$$
\left(\Omega_{j}(t), \Omega_{k}(t)\right)=\int_{-1}^{1} \Omega_{j}(t) \Omega_{k}(t)=h_{k} \delta_{j k}
$$


where $h_{i}=\frac{2}{2 i+1}$ and $\delta_{j k}$ is the Dirac function. Thus, for any $v \in L^{2}[-1,1]$,

$$
v(t)=\sum_{i=0}^{\infty} a_{i} \Omega_{i}(t), \quad a_{i}=\frac{1}{h_{i}} \int_{-1}^{1} v(t) \Omega_{i}(t) d t .
$$

Let $S_{N}[-1,1]$ be the set of all polynomials of degree at most $N(N \geq 0)$. Thus, for any $\varphi \in S_{2 N-1}[-1,1]$, we have

$$
\int_{-1}^{1} \varphi(t) d t=\sum_{i=0}^{N} \varpi_{N, i} \varphi\left(t_{N, i}\right)
$$

where $t_{N, k}(0 \leq k \leq N)$ and $\varpi_{N, k}(0 \leq k \leq N)$ denote the nodes and the Christoffel numbers of Legendre-Gauss-Lobatto (L-GL) interpolation on the classical interval $[-1,1]$.

The norm and the discrete inner product are defined as follows:

$$
\chi_{N}=(\chi, v)_{N}^{\frac{1}{2}},(\chi, v)_{N}=\sum_{j=0}^{N} \chi\left(t_{N, j}\right) v\left(t_{N, j}\right) \varpi_{N, j} .
$$

\section{Numerical simulations}

The aim of this paper is devoted to the development of a new scheme based on spectral collocation method for solving the fractional-order differential system of GBM-IS (1). The accuracy of the proposed methods is proved by convergence analysis. According to the rigorous mathematical proof provided by convergence analysis of fractional Legendre spectral collocation method [37-40] for differential equations, we can expect the spectral convergence rate for the fractional-order differential system of GBM-IS. Therefore, we can achieve very high accuracy by merely using a small number of fractional Legendre collocation nodes. We will discuss error analysis of our proposed methods to show that spectral convergence is obtained for sufficiently smooth solution functions.

In this paper, shifted fractional-order Legendre orthogonal functions (SFOLOF) in the interval $[0, \mathcal{L}]$ are outputted of the classical Legendre polynomial. Now, we define the SFOLOF outputted Legendre polynomial. The SFOLOF is offered by

$$
\Omega_{\mathcal{L}, j}^{(\varepsilon)}(t)=\Omega_{j}\left(2\left(\frac{x}{\mathcal{L}}\right)^{\varepsilon}-1\right), \quad 0<\varepsilon<1, j=0,1, \ldots, 0 \leq t \leq \mathcal{L} .
$$

Also, we list and derive some facts related to the SFOLOF like orthogonality, corresponding nodes, and Christoffel numbers of the shifted fractional-order LegendreGauss-Radau interpolation. For $\mathcal{W}_{\mathcal{L}, f}^{(\varepsilon)}(t)=t^{\varepsilon-1}$, the set of SFOLOF forms a complete $L_{\mathcal{W}_{\mathcal{L}, f}^{(\varepsilon)}}^{2}[0, \mathcal{L}]$-orthogonal system

$$
\int_{0}^{\mathcal{L}} \Omega_{\mathcal{L}, i}^{(\varepsilon)}(t) \Omega_{\mathcal{L}, j}^{(\varepsilon)}(t) \mathcal{W}_{\mathcal{L}, f}^{(\varepsilon)}(t) d x=\delta_{i j} h_{\mathcal{L}, k}^{(\varepsilon)}
$$

where $h_{\mathcal{L}, k}^{(\varepsilon)}=\frac{\mathcal{L}^{\varepsilon}}{2(2 k+1) \varepsilon}$.

Proof Based on the orthogonality property of Legendre polynomials, we have

$$
\int_{-1}^{1} \Omega_{r}(\xi) \Omega_{s}(\xi) d \xi=\frac{2}{2 r+1} \delta_{r s}
$$


Let $\xi=2\left(\frac{\zeta}{\mathcal{L}}\right)^{\varepsilon}-1$, we get

$$
\begin{aligned}
\int_{-1}^{1} \Omega_{r}(\xi) \Omega_{s}(\xi) d \xi & =\frac{2 \varepsilon}{\mathcal{L}^{\varepsilon}} \int_{0}^{\mathcal{L}} \zeta^{\varepsilon-1} \Omega_{r}\left(2\left(\frac{\zeta}{\mathcal{L}}\right)^{\varepsilon}-1\right) \Omega_{s}\left(2\left(\frac{\zeta}{\mathcal{L}}\right)^{\varepsilon}-1\right) d \zeta \\
& =\left(\frac{2}{\mathcal{L}^{\varepsilon}}\right) \int_{0}^{\mathcal{L}} \Omega_{\mathcal{L}, r}^{(\varepsilon)}(\zeta) \Omega_{\mathcal{L}, s}^{(\varepsilon)}(\zeta) d \zeta \\
& =\frac{2}{2 r+1} \delta_{r s} .
\end{aligned}
$$

Consequently, this yields

$$
\int_{0}^{\mathcal{L}} \Omega_{\mathcal{L}, r}^{(\varepsilon)}(\zeta) \Omega_{\mathcal{L}, s}^{(\varepsilon)}(\zeta) d \zeta=\delta_{r s} \frac{\mathcal{L}^{\varepsilon}}{2(2 r+1) \varepsilon}
$$

Let $\Omega_{\mathcal{M}}=\operatorname{span}\left\{\Omega_{\mathcal{L}, r}^{(\varepsilon)}: 0 \leq r \leq \mathcal{M}\right\}$ be the finite-dimensional fractional-polynomial space. Owing to the orthogonal property $(12)$, the function $\Theta(\xi) \in L_{\mathcal{W}_{f}^{(\varepsilon)}}^{2}[0, \mathcal{L}]$ may be extracted as follows:

$$
\Theta(\xi)=\sum_{r=0}^{\infty} \varrho_{r} \Omega_{\mathcal{L}, r}^{(\varepsilon)}(\xi), \quad \varrho_{r}=\frac{1}{h_{\mathcal{L}, r}^{(\varepsilon)}} \int_{0}^{\mathcal{L}} \Omega_{\mathcal{L}, i}^{(\varepsilon)}(\xi) \Theta(\xi) \mathcal{W}_{\mathcal{L}, f}^{(\varepsilon)}(\xi) d \xi
$$

The corresponding nodes and corresponding Christoffel numbers of the shifted fractional Legendre-Gauss (Gauss-Radau or Gauss-Lobatto) interpolation in the interval $[0, \mathcal{L}]$ can be given by

$$
t_{\mathcal{M}, s}^{\mathcal{L}, \varepsilon}=\mathcal{L}\left(\frac{t_{\mathcal{K}, s}+1}{2}\right)^{\frac{1}{\varepsilon}}, \quad \varpi_{\mathcal{L}, \mathcal{K}, s}^{(\varepsilon)}=\left(\frac{\mathcal{L}^{\varepsilon}}{2}\right) \varpi_{\mathcal{K}, s}, \quad 0 \leq s \leq \mathcal{M},
$$

where $x_{\mathcal{K}, s}$, and $\varpi_{\mathcal{K}, s}, 0 \leq s \leq \mathcal{M}$, are the nodes and the Christoffel numbers of the standard Legendre-Gauss (Gauss-Radau or Gauss-Lobatto) interpolation in the interval $[-1,1]$.

Proof Using Legendre-Gauss quadrature property, we have

$$
\begin{aligned}
\int_{0}^{\mathcal{L}} \mathcal{W}_{\mathcal{L}, f}^{(\varepsilon)}(\xi) \phi(\xi) d \xi & =\int_{0}^{\mathcal{L}} \xi^{\varepsilon-1} \phi(\xi) d \xi \\
& =\left(\frac{\mathcal{L}^{\varepsilon}}{2 \varepsilon}\right) \int_{-1}^{1} \phi\left(\mathcal{L}\left(\frac{\zeta+1}{2}\right)^{\frac{1}{\varepsilon}}\right) d \zeta \\
& =\left(\frac{\mathcal{L}^{\varepsilon}}{2 \varepsilon}\right) \sum_{j=0}^{\mathcal{M}} \varpi_{\mathcal{M}, j} \phi\left(\mathcal{L}\left(\frac{\zeta_{\mathcal{M}, j}+1}{2}\right)^{\frac{1}{\varepsilon}}\right) \\
& =\sum_{j=0}^{\mathcal{M}} \varpi_{\mathcal{L}, \mathcal{M}, j}^{(\varepsilon)} \phi\left(\xi_{\mathcal{L}, \mathcal{M}, j}^{(\varepsilon)}\right) .
\end{aligned}
$$

The proof is completed. 
The solution of Eq. (1) is approximated as follows:

$$
\begin{array}{ll}
S_{\mathcal{M}, \varepsilon}(t)=\sum_{j=0}^{\mathcal{M}} \varsigma_{j} \Omega_{\mathcal{L}, j}^{\varepsilon}(t), & R_{\mathcal{M}, \varepsilon}(t)=\sum_{j=0}^{\mathcal{M}} \rho_{j} \Omega_{\mathcal{L}, j}^{\varepsilon}(t), \\
N_{\mathcal{M}, \varepsilon}(t)=\sum_{j=0}^{\mathcal{M}} \kappa_{j} \Omega_{\mathcal{L}, j}^{\varepsilon}(t), & Z_{\mathcal{M}, \varepsilon}(t)=\sum_{j=0}^{\mathcal{M}} \sigma_{j} \Omega_{\mathcal{L}, j}^{\varepsilon}(t) .
\end{array}
$$

Moreover, the Caputo fractional derivative of any order $\mu$ of the shifted fractional-order Legendre orthogonal function is derived. The Caputo fractional derivative of order $\mu$ is defined as follows:

$$
D^{\mu} \chi(t)=\frac{1}{\Gamma(m-\mu)} \int_{0}^{t}(t-\eta)^{m-\mu-1} \frac{d^{m} \chi(\eta)}{d \eta^{m}} d \eta, \quad m-1<\mu \leq m, t>0,
$$

where $m$ is the ceiling function of $\mu$. The analytical form of $\Omega_{\mathcal{L}, j}^{(\varepsilon)}(t)$ is given by

$$
\Omega_{\mathcal{L}, j}^{(\varepsilon)}(t)=\sum_{k=0}^{j} E_{k}^{(\varepsilon, j)} t^{\varepsilon k}
$$

where

$$
E_{k}^{(\varepsilon, j)}=\frac{(-1)^{j-k}(\Gamma(j+k+1))}{(j-k) !(\Gamma(k+1))^{2} \mathcal{L}^{\varepsilon k}}
$$

By means of Eq. (15), we find

$$
D_{t}^{\mu}\left(t^{\varepsilon k}\right)=\frac{k \varepsilon \Gamma(k \varepsilon) t^{k \varepsilon-\mu}}{\Gamma(k \varepsilon-\mu+1)}, \quad \forall k, \text { where } k \varepsilon>\mu,
$$

thus

$$
\begin{aligned}
D_{t}^{\mu}\left(\Omega_{\mathcal{L}, j}^{(\varepsilon)}(t)\right) & =\Omega_{j}^{(\mu, \varepsilon)}(t) \\
& =\sum_{k=1}^{j} E_{k}^{(\varepsilon, j)} \frac{k \varepsilon \Gamma(k \varepsilon) t^{k \varepsilon-\mu}}{\Gamma(k \varepsilon-\mu+1)} .
\end{aligned}
$$

The Caputo fractional derivative of order $\mu$ of the approximate solution is then estimated as follows:

$$
\begin{array}{ll}
D^{\mu} S_{\mathcal{M}, \varepsilon}(t)=\sum_{j=0}^{\mathcal{M}} \varsigma_{j} \Omega_{\mathcal{L}, j}^{\mu, \varepsilon}(t), & D^{\mu} R_{\mathcal{M}, \varepsilon}(t)=\sum_{j=0}^{\mathcal{M}} \rho_{j} \Omega_{\mathcal{L}, j}^{\mu, \varepsilon}(t), \\
D^{\mu} N_{\mathcal{M}, \varepsilon}(t)=\sum_{j=0}^{\mathcal{M}} \kappa_{j} \Omega_{\mathcal{L}, j}^{\mu, \varepsilon}(t), & D^{\mu} Z_{\mathcal{M}, \varepsilon}(t)=\sum_{j=0}^{\mathcal{M}} \sigma_{j} \Omega_{\mathcal{L}, j}^{\mu, \varepsilon}(t) .
\end{array}
$$

Spectral collocation technique is addressed to solve the previous problem subject to the initial conditions. Firstly, a collocation method based on fractional-order Legendre basis 
functions is introduced for the fractional-order differential system of GBM-IS with initial conditions. Consequently, we obtain a system of algebraic equations. Based on the above details, we obtain

$$
\begin{aligned}
& \sum_{j=0}^{\mathcal{M}} \varsigma_{j} \Omega_{\mathcal{L}, j}^{\mu, \varepsilon}(t) \\
& =p S(t)+r_{1} \sum_{j=0}^{\mathcal{M}} \varsigma_{j} \Omega_{\mathcal{L}, j}^{\varepsilon}(t)\left(k_{1}-\beta_{1} \sum_{j=0}^{\mathcal{M}} \varsigma_{j} \Omega_{\mathcal{L}, j}^{\varepsilon}(t)\right)-\gamma \sum_{j=0}^{\mathcal{M}} \varsigma_{j} \Omega_{\mathcal{L}, j}^{\varepsilon}(t) \sum_{j=0}^{\mathcal{M}} \rho_{j} \Omega_{\mathcal{L}, j}^{\varepsilon}(t) \\
& -d_{1} \sum_{j=0}^{\mathcal{M}} \varsigma_{j} \Omega_{\mathcal{L}, j}^{\varepsilon}(t)-\tau_{1} \sum_{j=0}^{\mathcal{M}} \varsigma_{j} \Omega_{\mathcal{L}, j}^{\varepsilon}(t) \sum_{j=0}^{\mathcal{M}} \kappa_{j} \Omega_{\mathcal{L}, j}^{\varepsilon}(t), \\
& \sum_{j=0}^{\mathcal{M}} \rho_{j} \Omega_{\mathcal{L}, j}^{\mu, \varepsilon}(t) \\
& =r_{2} \sum_{j=0}^{\mathcal{M}} \rho_{j} \Omega_{\mathcal{L}, j}^{\varepsilon}(t)\left(k_{2}-\beta_{2} \sum_{j=0}^{\mathcal{M}} \rho_{j} \Omega_{\mathcal{L}, j}^{\varepsilon}(t)\right)+\gamma \sum_{j=0}^{\mathcal{M}} \varsigma_{j} \Omega_{\mathcal{L}, j}^{\varepsilon}(t) \sum_{j=0}^{\mathcal{M}} \rho_{j} \Omega_{\mathcal{L}, j}^{\varepsilon}(t) \\
& -d_{2} \sum_{j=0}^{\mathcal{M}} \rho_{j} \Omega_{\mathcal{L}, j}^{\varepsilon}(t)-\tau_{2} \sum_{j=0}^{\mathcal{M}} \rho_{j} \Omega_{\mathcal{L}, j}^{\varepsilon}(t) \sum_{j=0}^{\mathcal{M}} \kappa_{j} \Omega_{\mathcal{L}, j}^{\varepsilon}(t), \\
& \sum_{j=0}^{\mathcal{M}} \kappa_{j} \Omega_{\mathcal{L}, j}^{\mu, \varepsilon}(t) \\
& =\delta \sum_{j=0}^{\mathcal{M}} \kappa_{j} \Omega_{\mathcal{L}, j}^{\varepsilon}(t) \sum_{j=0}^{\mathcal{M}} \sigma_{j} \Omega_{\mathcal{L}, j}^{\varepsilon}(t)-\epsilon \sum_{j=0}^{\mathcal{M}} \sigma_{j} \Omega_{\mathcal{L}, j}^{\varepsilon}(t) \sum_{j=0}^{\mathcal{M}} \kappa_{j} \Omega_{\mathcal{L}, j}^{\varepsilon}(t)-d_{3} \sum_{j=0}^{\mathcal{M}} \kappa_{j} \Omega_{\mathcal{L}, j}^{\varepsilon}(t), \\
& \sum_{j=0}^{\mathcal{M}} \sigma_{j} \Omega_{\mathcal{L}, j}^{\mu, \varepsilon}(t) \\
& =r_{3} \sum_{j=0}^{\mathcal{M}} \sigma_{j} \Omega_{\mathcal{L}, j}^{\varepsilon}(t)\left(k_{3}-\beta_{3} \sum_{j=0}^{\mathcal{M}} \sigma_{j} \Omega_{\mathcal{L}, j}^{\varepsilon}(t)\right)-\delta \sum_{j=0}^{\mathcal{M}} \kappa_{j} \Omega_{\mathcal{L}, j}^{\varepsilon}(t) \sum_{j=0}^{\mathcal{M}} \sigma_{j} \Omega_{\mathcal{L}, j}^{\varepsilon}(t) \\
& +\epsilon \sum_{j=0}^{\mathcal{M}} \sigma_{j} \Omega_{\mathcal{L}, j}^{\varepsilon}(t) \sum_{j=0}^{\mathcal{M}} \kappa_{j} \Omega_{\mathcal{L}, j}^{\varepsilon}(t)-d_{4} \sum_{j=0}^{\mathcal{M}} \sigma_{j} \Omega_{\mathcal{L}, j}^{\varepsilon}(t)
\end{aligned}
$$

Using the initial conditions, we obtain

$$
\begin{aligned}
& \sum_{j=0}^{\mathcal{M}} \varsigma_{j} \Omega_{\mathcal{L}, j}^{\varepsilon}(0)=S_{0}, \quad \sum_{j=0}^{\mathcal{M}} \rho_{j} \Omega_{\mathcal{L}, j}^{\varepsilon}(0)=R_{0}, \\
& \sum_{j=0}^{\mathcal{M}} \kappa_{j} \Omega_{\mathcal{L}, j}^{\varepsilon}(0)=N_{0}, \quad \sum_{j=0}^{\mathcal{M}} \sigma_{j} \Omega_{\mathcal{L}, j}^{\varepsilon}(0)=Z_{0} .
\end{aligned}
$$


In the proposed method, the residual of (17) is set to zero at the $\mathcal{M}$ points

$$
\begin{aligned}
& \sum_{j=0}^{\mathcal{M}} \varsigma_{j} \Omega_{\mathcal{L}, j}^{\mu, \varepsilon}\left(t_{\mathcal{M}, s}^{\mathcal{L}, \varepsilon}\right) \\
& =p S\left(t_{\mathcal{M}, s}^{\mathcal{L}, \varepsilon}\right)+r_{1} \sum_{j=0}^{\mathcal{M}} \varsigma_{j} \Omega_{\mathcal{L}, j}^{\varepsilon}\left(t_{\mathcal{M}, s}^{\mathcal{L}, \varepsilon}\right)\left(k_{1}-\beta_{1} \sum_{j=0}^{\mathcal{M}} \varsigma_{j} \Omega_{\mathcal{L}, j}^{\varepsilon}\left(t_{\mathcal{M}, s}^{\mathcal{L}, \varepsilon}\right)\right)-d_{1} \sum_{j=0}^{\mathcal{M}} \varsigma_{j} \Omega_{\mathcal{L}, j}^{\varepsilon}\left(t_{\mathcal{M}, s}^{\mathcal{L}, \varepsilon}\right) \\
& -\gamma \sum_{j=0}^{\mathcal{M}} \varsigma_{j} \Omega_{\mathcal{L}, j}^{\varepsilon}\left(t_{\mathcal{M}, s}^{\mathcal{L}, \varepsilon}\right) \sum_{j=0}^{\mathcal{M}} \rho_{j} \Omega_{\mathcal{L}, j}^{\varepsilon}\left(t_{\mathcal{M}, s}^{\mathcal{L}, \varepsilon}\right)-\tau_{1} \sum_{j=0}^{\mathcal{M}} \varsigma_{j} \Omega_{\mathcal{L}, j}^{\varepsilon}\left(t_{\mathcal{M}, s}^{\mathcal{L}, \varepsilon}\right) \sum_{j=0}^{\mathcal{M}} \kappa_{j} \Omega_{\mathcal{L}, j}^{\varepsilon}\left(t_{\mathcal{M}, s}^{\mathcal{L}, \varepsilon}\right), \\
& \sum_{j=0}^{\mathcal{M}} \rho_{j} \Omega_{\mathcal{L}, j}^{\mu, \varepsilon}\left(t_{\mathcal{M}, s}^{\mathcal{L}, \varepsilon}\right) \\
& =r_{2} \sum_{j=0}^{\mathcal{M}} \rho_{j} \Omega_{\mathcal{L}, j}^{\varepsilon}\left(t_{\mathcal{M}, s}^{\mathcal{L}, \varepsilon}\right)\left(k_{2}-\beta_{2} \sum_{j=0}^{\mathcal{M}} \rho_{j} \Omega_{\mathcal{L}, j}^{\varepsilon}\left(t_{\mathcal{M}, s}^{\mathcal{L}, \varepsilon}\right)\right)-d_{2} \sum_{j=0}^{\mathcal{M}} \rho_{j} \Omega_{\mathcal{L}, j}^{\varepsilon}\left(t_{\mathcal{M}, s}^{\mathcal{L}, \varepsilon}\right) \\
& +\gamma \sum_{j=0}^{\mathcal{M}} \varsigma_{j} \Omega_{\mathcal{L}, j}^{\varepsilon}\left(t_{\mathcal{M}, s}^{\mathcal{L}, \varepsilon}\right) \sum_{j=0}^{\mathcal{M}} \rho_{j} \Omega_{\mathcal{L}, j}^{\varepsilon}\left(t_{\mathcal{M}, s}^{\mathcal{L}, \varepsilon}\right) \\
& -\tau_{2} \sum_{j=0}^{\mathcal{M}} \rho_{j} \Omega_{\mathcal{L}, j}^{\varepsilon}\left(t_{\mathcal{M}, s}^{\mathcal{L}, \varepsilon}\right) \sum_{j=0}^{\mathcal{M}} \kappa_{j} \Omega_{\mathcal{L}, j}^{\varepsilon}\left(t_{\mathcal{M}, s}^{\mathcal{L}, \varepsilon}\right) \\
& \sum_{j=0}^{\mathcal{M}} \kappa_{j} \Omega_{\mathcal{L}, j}^{\mu, \varepsilon}\left(t_{\mathcal{M}, s}^{\mathcal{L}, \varepsilon}\right) \\
& =\delta \sum_{j=0}^{\mathcal{M}} \kappa_{j} \Omega_{\mathcal{L}, j}^{\varepsilon}\left(t_{\mathcal{M}, s}^{\mathcal{L}, \varepsilon}\right) \sum_{j=0}^{\mathcal{M}} \sigma_{j} \Omega_{\mathcal{L}, j}^{\varepsilon}\left(t_{\mathcal{M}, s}^{\mathcal{L}, \varepsilon}\right)-\epsilon \sum_{j=0}^{\mathcal{M}} \sigma_{j} \Omega_{\mathcal{L}, j}^{\varepsilon}\left(t_{\mathcal{M}, s}^{\mathcal{L}, \varepsilon}\right) \sum_{j=0}^{\mathcal{M}} \kappa_{j} \Omega_{\mathcal{L}, j}^{\varepsilon}\left(t_{\mathcal{M}, s}^{\mathcal{L}, \varepsilon}\right) \\
& -d_{3} \sum_{j=0}^{\mathcal{M}} \kappa_{j} \Omega_{\mathcal{L}, j}^{\varepsilon}\left(t_{\mathcal{M}, s}^{\mathcal{L}, \varepsilon}\right) \\
& \sum_{j=0}^{\mathcal{M}} \sigma_{j} \Omega_{\mathcal{L}, j}^{\mu, \varepsilon}\left(t_{\mathcal{M}, s}^{\mathcal{L}, \varepsilon}\right) \\
& =r_{3} \sum_{j=0}^{\mathcal{M}} \sigma_{j} \Omega_{\mathcal{L}, j}^{\varepsilon}\left(t_{\mathcal{M}, s}^{\mathcal{L}, \varepsilon}\right)\left(k_{3}-\beta_{3} \sum_{j=0}^{\mathcal{M}} \sigma_{j} \Omega_{\mathcal{L}, j}^{\varepsilon}\left(t_{\mathcal{M}, s}^{\mathcal{L}, \varepsilon}\right)\right)-d_{4} \sum_{j=0}^{\mathcal{M}} \sigma_{j} \Omega_{\mathcal{L}, j}^{\varepsilon}\left(t_{\mathcal{M}, s}^{\mathcal{L}, \varepsilon}\right) \\
& -\delta \sum_{j=0}^{\mathcal{M}} \kappa_{j} \Omega_{\mathcal{L}, j}^{\varepsilon}\left(t_{\mathcal{M}, s}^{\mathcal{L}, \varepsilon}\right) \sum_{j=0}^{\mathcal{M}} \sigma_{j} \Omega_{\mathcal{L}, j}^{\varepsilon}\left(t_{\mathcal{M}, s}^{\mathcal{L}, \varepsilon}\right)+\epsilon \sum_{j=0}^{\mathcal{M}} \sigma_{j} \Omega_{\mathcal{L}, j}^{\varepsilon}\left(t_{\mathcal{M}, s}^{\mathcal{L}, \varepsilon}\right) \sum_{j=0}^{\mathcal{M}} \kappa_{j} \Omega_{\mathcal{L}^{\prime}, j}^{\varepsilon}\left(t_{\mathcal{M}, s}^{\mathcal{L}, \varepsilon}\right) .
\end{aligned}
$$

Taking $s=1,2, \ldots, \mathcal{M}$, we get a system of $(4 \mathcal{M}+4)$ algebraic equations which can be solved for the unknown coefficients. The rigor of the novel method is demonstrated with several test problems. We can indicate that our numerical method can also accommodate other methods. For example, the spectral Tau approach for nonsmooth temporal solution may be fallen apart. Employing fractional-order Legendre functions instead of the classical Legendre functions stopped this deterioration. Suppose that $D^{k \varepsilon} \mathcal{Z}(t) \in C[0, \mathcal{L}]$ for $k=$ $0,1, \ldots, \mathcal{M}$. If $\mathcal{Z}_{\mathcal{M}, \varepsilon}(t)$ is the best approximation to $\mathcal{Z}(t)$ from $\Omega_{\mathcal{M}}$, then the error bound 
is presented as follows:

$$
\left\|\mathcal{Z}(t)-\mathcal{Z}_{\mathcal{M}, \varepsilon}(t)\right\|_{\mathcal{W}_{\mathcal{L}, f}^{(\varepsilon)}(t)} \leq \frac{\sqrt{\mathcal{L}^{\varepsilon(2 \mathcal{M}+3)}} E_{\varepsilon}}{(\Gamma((\mathcal{M}+1) \varepsilon+1))}
$$

Proof. Since $\mathcal{Z}_{\mathcal{M}, \varepsilon}(t)$ is the best approximation to $\mathcal{Z}(t)$ from $\Omega_{\mathcal{M}}$, then by the definition of the best approximation, we have

$$
\forall \mathcal{V}_{\mathcal{M}}(t) \in \Omega_{\mathcal{M}}, \quad\left\|\mathcal{Z}(t)-\mathcal{Z}_{\mathcal{M}, \varepsilon}(t)\right\|_{\mathcal{W}_{\mathcal{L}_{f} f}^{(\varepsilon)}(t)} \leq\left\|\mathcal{Z}(t)-\mathcal{V}_{\mathcal{M}}(t)\right\|_{\mathcal{W}_{\mathcal{L}_{f} f}^{(\varepsilon)}(t)}
$$

Based on the generalized Taylors formula [41], we obtain $\mathcal{V}_{\mathcal{M}}(t)=\sum_{k=0}^{\mathcal{M}} \frac{t^{k \varepsilon}}{\Gamma(k \varepsilon+1)} D^{k \varepsilon} \mathcal{Z}\left(0^{+}\right)$. Thus, an upper bound of the absolute error is

$$
\left|\mathcal{Z}(t)-\sum_{k=0}^{\mathcal{M}} \frac{t^{k \varepsilon}}{\Gamma(k \varepsilon+1)} D^{k \varepsilon} \mathcal{Z}\left(0^{+}\right)\right| \leq E_{\varepsilon} \frac{t^{(\mathcal{M}+1) \varepsilon}}{\Gamma((\mathcal{M}+1) \varepsilon+1)}
$$

where $E_{\varepsilon}=\operatorname{MAX}\left\{D^{k \varepsilon} \mathcal{Z}(\xi), \xi \in[0, \mathcal{L}]\right\}$. Then we conclude that the following

$$
\begin{aligned}
\left\|\mathcal{Z}(t)-\mathcal{Z}_{\mathcal{M}, \varepsilon}(t)\right\|_{\mathcal{W}_{\mathcal{L}, f}^{(\varepsilon)}(t)}^{2} & \leq\left\|\mathcal{Z}(t)-\sum_{k=0}^{\mathcal{M}} \frac{t^{k \varepsilon}}{\Gamma(k \varepsilon+1)} D^{k \varepsilon} \mathcal{Z}\left(0^{+}\right)\right\|_{\mathcal{W}_{\mathcal{L}, f}^{(\varepsilon)}(t)}^{2} \\
& \leq \frac{E_{\varepsilon}^{2}}{(\Gamma((\mathcal{M}+1) \varepsilon+1))^{2}} \int_{0}^{\mathcal{L}} t^{2(\mathcal{M}+1) \varepsilon} \mathcal{W}_{\mathcal{L}, f}^{(\varepsilon)}(t) d x \\
& \leq \frac{E_{\varepsilon}^{2}}{(\Gamma((\mathcal{M}+1) \varepsilon+1))^{2}} \int_{0}^{\mathcal{L}} t^{2(\mathcal{M}+1) \varepsilon} t^{\varepsilon-1} d x \\
& \leq \frac{\mathcal{L}^{\varepsilon(2 \mathcal{M}+3)} E_{\varepsilon}^{2}}{(\Gamma((\mathcal{M}+1) \varepsilon+1))^{2}} \int_{0}^{1} \xi^{2 \mathcal{M}+\varepsilon+1} \xi d \xi \\
& \leq \frac{\mathcal{L}^{\varepsilon(2 \mathcal{M}+3)} E_{\varepsilon}^{2}}{(\Gamma((\mathcal{M}+1) \varepsilon+1))^{2}}
\end{aligned}
$$

is obtained for the approximate solutions. The convergence of the proposed method depends basically on the above error bound.

\section{Numerical results}

Using the algorithm presented in the previous section, we give in this section some numerical results. We discuss the fractional-order differential system of a GBM-IS interaction.

Example 1 Let us consider the parameter values $\mathcal{M}=20, \mathcal{L}=15, \varepsilon=1, \mu=0.9, p=0.192$, $r_{1}=0.5, k_{1}=4.704, \beta_{1}=0.75, \gamma=\frac{1}{10^{2}}, d_{1}=0.6, \tau_{1}=0.3, r_{2}=0.525, k_{2}=1.232, \beta_{2}=0.1$, $d_{2}=0.006, \tau_{2}=0.03, \delta=0.201, \epsilon=0.01, d_{3}=0.07, r_{3}=0.0146, k_{3}=0.15, \delta_{1}=0.06, d_{4}=$ 0.07 . For the given parameter values, we plot in Fig. 1 the numerical solution curves of the fractional-order differential system of GBM-IS. Moreover, we obtain the numerical solutions of the fractional-order differential system of GBM-IS as follows:

$$
\begin{aligned}
& S_{20,1}(t) \\
& \quad=6.819 \times 10^{-15} t^{20}-1.1283 \times 10^{-12} t^{19}+8.606 \times 10^{-11} t^{18}-4.017 \times 10^{-9} t^{17}
\end{aligned}
$$



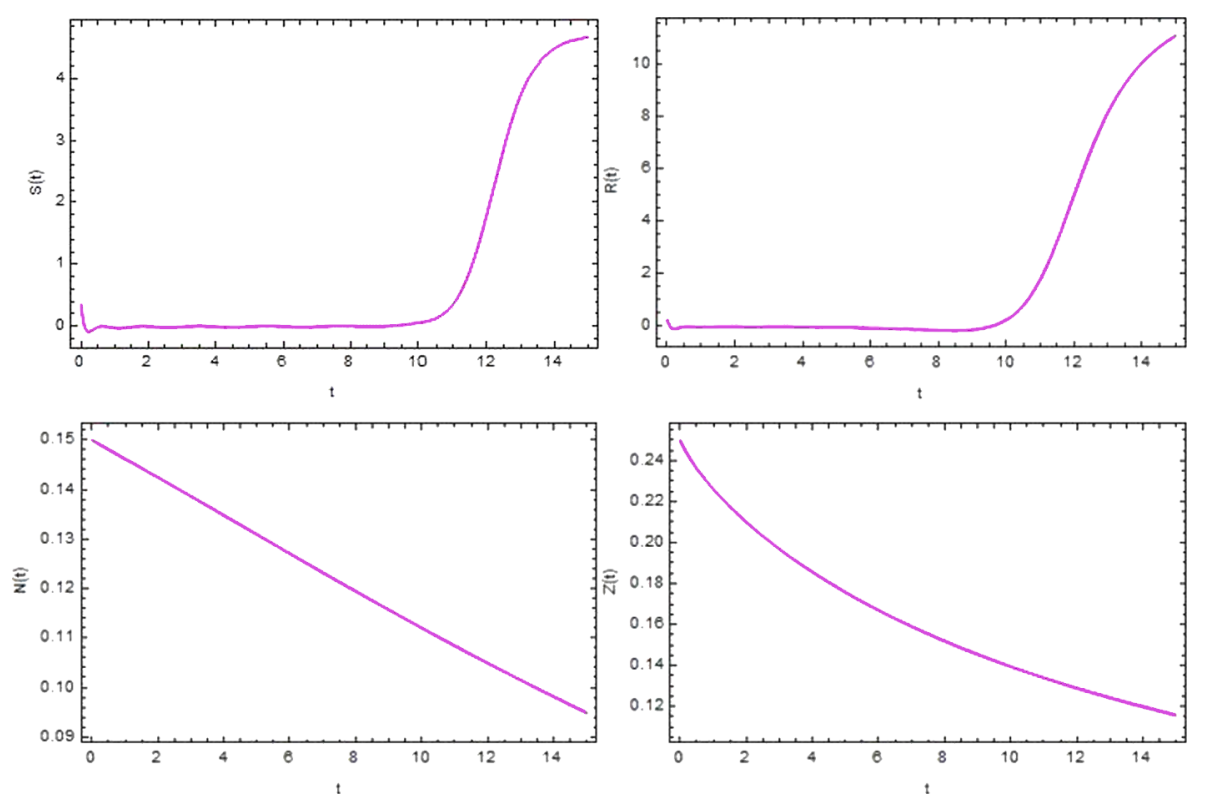

Figure 1 Numerical solution curves of the fractional-order differential system of GBM-IS

$+1.285 \times 10^{-7} t^{16}-2.988 \times 10^{-6} t^{15}+0.0000522623 t^{14}-7.023 \times 10^{-4} t^{13}$

$+7.338 \times 10^{-3} t^{12}$

$-5.998 \times 10^{-2} t^{11}+0.384 t^{10}-1.914 t^{9}+7.379 t^{8}-21.687 t^{7}+47.604 t^{6}$

$-75.792 t^{5}+83.923 t^{4}-60.721 t^{3}+25.996 t^{2}-5.493 x+0.35$,

$R_{20,1}(t)$

$=1.590 \times 10^{-14} t^{20}-2.439 \times 10^{-12} t^{19}+1.732 \times 10^{-10} t^{18}-7.559 \times 10^{-9} t^{17}$

$+2.268 \times 10^{-7} t^{16}-4.961 \times 10^{-6} t^{15}+8.188 \times 10^{-5} t^{14}-1.041 \times 10^{-3} t^{13}$

$+1.031 \times 10^{-2} t^{12}$

$-8.010 \times 10^{-2} t^{11}+0.488 t^{10}-2.320 t^{9}+8.543 t^{8}-24.006 t^{7}+50.418 t^{6}$

$-76.836 t^{5}+81.431 t^{4}-56.344 t^{3}+23.013 t^{2}-4.603 t+0.25$,

$N_{20,1}(t)$

$$
\begin{aligned}
= & .288 \times 10^{-20} t^{20}-2.142 \times 10^{-18} t^{19}+1.660 \times 10^{-16} t^{18}-7.956 \times 10^{-15} t^{17} \\
+ & 2.642 \times 10^{-13} t^{16}-6.453 \times 10^{-12} t^{15}+1.200 \times 10^{-10} t^{14}-1.738 \times 10^{-9} t^{13} \\
+ & 1.984 \times 10^{-8} t^{12} \\
& -1.801 \times 10^{-7} t^{11}+1.302 \times 10^{-6} t^{10}-7.496 \times 10^{-6} t^{9}+3.418 \times 10^{-5} t^{8} \\
& -1.224 \times 10^{-4} t^{7} \\
+ & 3.401 \times 10^{-4} t^{6}-7.209 \times 10^{-4} t^{5}+1.142 \times 10^{-3} t^{4}-1.313 \times 10^{-3} t^{3} \\
+ & 1.007 t^{2}-4.162 t+0.15,
\end{aligned}
$$




$$
\begin{aligned}
Z_{20,1}( & t) \\
= & 4.988 \times 10^{-19} t^{20}-8.177 \times 10^{-17} t^{19}+6.243 \times 10^{-15} t^{18}-2.946 \times 10^{-13} t^{17} \\
& +9.627 \times 10^{-12} t^{16}-2.311 \times 10^{-10} t^{15}+4.222 \times 10^{-9} t^{14}-5.997 \times 10^{-8} t^{13} \\
& +6.711 \times 10^{-7} t^{12} \\
& -5.960 \times 10^{-6} t^{11}+4.212 \times 10^{-4} t^{10}-2.365 \times 10^{-3} t^{9}+1.050 \times 10^{-3} t^{8} \\
& -3.653 \times 10^{-3} t^{7} \\
& +9.833 \times 10^{-3} t^{6}-2.014 \times 10^{-2} t^{5}+3.080 \times 10^{-2} t^{4}-3.464 \times 10^{-2} t^{3} \\
& +3.010 \times 10^{-2} t^{2}-3.708 \times 10^{-2} t+0.25 .
\end{aligned}
$$

Example 2 Let us consider the parameter values $\mathcal{M}=20, \mathcal{L}=10, \varepsilon=1, \mu=0.98, p=$ $0.192, r_{1}=0.5, k_{1}=4.704, \beta_{1}=0.75, \gamma=\frac{1}{10^{2}}, d_{1}=0.6, \tau_{1}=0.3, r_{2}=0.525, k_{2}=1.232, \beta_{2}=$ $0.1, d_{2}=0.006, \tau_{2}=0.03, \delta=0.201, \epsilon=0.01, d_{3}=0.07, r_{3}=0.5, k_{3}=1.17, \delta_{1}=0.06, d_{4}=$ 0.07. For the given parameter values, we plot in Fig. 2 the numerical solution curves of the fractional-order differential system of GBM-IS. Moreover, we obtain the numerical solutions of the fractional-order differential system of GBM-IS as follows:

$$
\begin{aligned}
S_{20,1}( & t) \\
= & 0.35-7.095 t+49.049 t^{2}-171.419 t^{3}+358.134 t^{4}-491.938 t^{5}+472.1 t^{6} \\
& -329.862 t^{7} \\
& +172.723 t^{8}-69.1681 t^{9}+21.479 t^{10}-5.217 t^{11}+0.995 t^{12}-0.149 t^{13} \\
& +1.740 \times 10^{-2} t^{14} \\
& -1.56649 \times 10^{-3} t^{15}+1.065 \times 10^{-4} t^{16}-5.286 \times 10^{-6} t^{17}+1.805 \times 10^{-7} t^{18} \\
& -3.789 \times 10^{-9} t^{19}+3.686 \times 10^{-11} t^{20}, \\
R_{20,1} & (t) \\
= & 0.25+0.110 t+0.398012 t^{2}-1.24704 t^{3}+2.64061 t^{4}-3.61478 t^{5}+3.43703 t^{6} \\
& -2.36652 t^{7} \\
& +1.2151 t^{8}-0.475 t^{9}+0.143 t^{10}-3.366 \times 10^{-2} t^{11}+6.180 \times 10^{-3} t^{12} \\
& -8.863 \times 10^{-4} t^{13}
\end{aligned}
$$

Figure 2 Curves of numerical solutions of the fractional-order differential system of GBM-IS

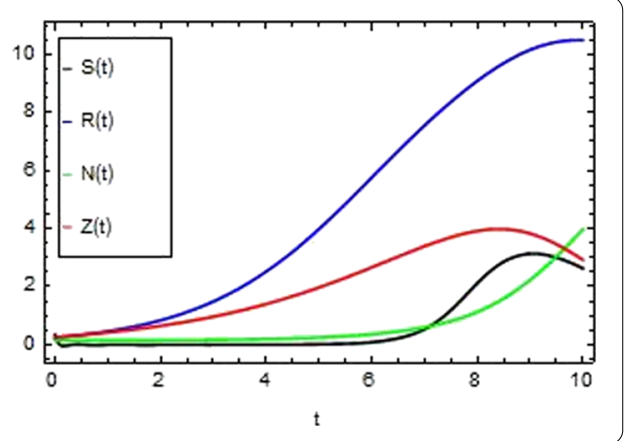




$$
\begin{aligned}
& +9.869 \times 10^{-5} t^{14}-8.430 \times 10^{-6} t^{15}+5.408 \times 10^{-7} t^{16}-2.517 \times 10^{-8} t^{17} \\
& +8.012 \times 10^{-10} t^{18} \\
& -1.557 \times 10^{-11} t^{19}+1.391 \times 10^{-13} t^{20}
\end{aligned}
$$

$N_{20,1}(t)$

$$
\begin{aligned}
= & 0.15-4.389 \times 10^{-3} t+9.869 \times 10^{-3} t^{2}-2.554 \times 10^{-2} t^{3}+5.355 \times 10^{-2} t^{4} \\
& -7.46 \times 10^{-2} t^{5} \\
& +7.315 \times 10^{-2} t^{6}-5.235 \times 10^{-2} t^{7}+2.811 \times 10^{-2} t^{8}-1.155 \times 10^{-2} t^{9} \\
& +3.684 \times 10^{-3} t^{10} \\
& -9.191 \times 10^{-4} t^{11}+1.801 \times 10^{-4} t^{12}-2.769 \times 10^{-5} t^{13}+3.3225 \times 10^{-6} t^{14} \\
& -3.073 \times 10^{-7} t^{15} \\
& +2.146 \times 10^{-8} t^{16}-1.093 \times 10^{-9} t^{17}+3.828 \times 10^{-11} t^{19}-8.234 \times 10^{-13} t^{18} \\
& +8.196 \times 10^{-15} t^{20}, \\
Z_{20,1} & (t) \\
= & 0.25+0.204 t-0.160 t^{2}+0.356 t^{3}-0.493 t^{4}+0.504 t^{5}-0.386 t^{6}+0.226 t^{7} \\
& -0.103 t^{8} \\
& +3.669 \times 10^{-2} t^{9}-1.039 \times 10^{-2} t^{10}+2.345 \times 10^{-3} t^{11}-4.217 \times 10^{-4} t^{12} \\
& +6.025 \times 10^{-5} t^{13} \\
& -6.786 \times 10^{-6} t^{14}+5.943 \times 10^{-7} t^{15}-3.959 \times 10^{-8} t^{16}+1.936 \times 10^{-9} t^{17} \\
& -6.547 \times 10^{-11} t^{18}+1.367 \times 10^{-12} t^{19}-1.325 \times 10^{-14} t^{20} .
\end{aligned}
$$

Let us consider the parameter values $\mathcal{M}=20, \mathcal{L}=10, \varepsilon=\frac{1}{2}, \mu=0.98, p=0.192, r_{1}=0.5$, $k_{1}=4.704, \beta_{1}=0.75, \gamma=\frac{1}{10^{2}}, d_{1}=0.6, \tau_{1}=0.3, r_{2}=0.525, k_{2}=1.232, \beta_{2}=0.1, d_{2}=0.006$, $\tau_{2}=0.03, \delta=0.201, \epsilon=0.01, d_{3}=0.07, r_{3}=0.5, k_{3}=1.17, \delta_{1}=0.06, d_{4}=0.07$. For the given parameter values, we plot in Fig. 3 the numerical solution curves of the fractionalorder differential system of GBM-IS. Moreover, we obtain the numerical solutions of the

Figure 3 Curves of numerical solutions of the fractional-order differential system of GBM-IS

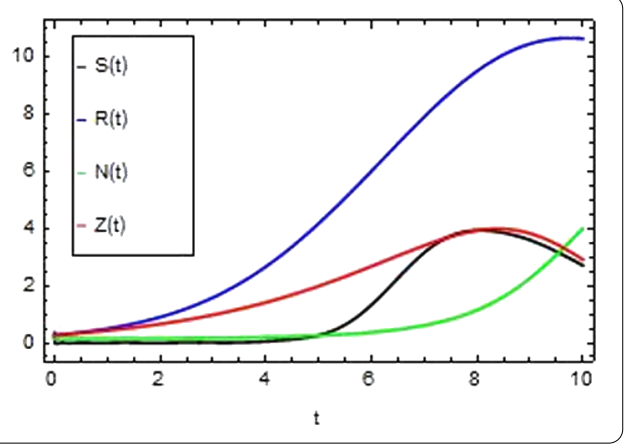


fractional-order differential system of GBM-IS as follows:

$$
\begin{aligned}
& S_{20, \frac{1}{2}}(t) \\
& =-6483.74 t^{\frac{3}{2}}-190,389 t^{\frac{5}{2}}-1.27907 \times 10^{6} t^{\frac{7}{2}}-2.64196 \times 10^{6} t^{\frac{9}{2}} \\
& -1.93473 \times 10^{6} t^{\frac{11}{2}} \\
& -529,435 t^{\frac{13}{2}}-52,742.2 t^{\frac{15}{2}}-1667.45 t^{\frac{17}{2}}-11.083 t^{\frac{19}{2}}+0.327 t^{10}+173.597 t^{9} \\
& +10,992.5 t^{8} \\
& +190,582 t^{7}+1.144 \times 10^{6} t^{6}+2.56069 \times 10^{6} t^{5}+2.10614 \times 10^{6} t^{4}+579,683 t^{3} \\
& +43,460.8 t^{2}+573.892 t-25.329 \sqrt{t}+0.35 \\
& R_{20, \frac{1}{2}}(t) \\
& =270.081 t^{\frac{3}{2}}+8184.07 t^{\frac{5}{2}}+57,358.4 t^{\frac{7}{2}}+124,777 t^{\frac{9}{2}}+97,060.2 t^{\frac{11}{2}}+28,435.1 t^{\frac{13}{2}} \\
& +3055.22 t^{\frac{15}{2}} \\
& +104.93 t^{\frac{17}{2}}+0.763 t^{\frac{19}{2}}-2.361 \times 10^{-2} t^{10}-11.4167 t^{9}-663.094 t^{8} \\
& -10,620.7 t^{7}-59,337.8 t^{6} \\
& -124,517 . t^{5}-96,817.7 t^{4}-25,418.7 t^{3}-1836.34 t^{2}-23.4701 t \\
& +1.03656 \sqrt{t}+0.25 \\
& N_{20, \frac{1}{2}}(t) \\
& =15.8473 t^{\frac{3}{2}}+478.632 t^{\frac{5}{2}}+3331.99 t^{\frac{7}{2}}+7166.34 t^{\frac{9}{2}}+5479.89 t^{\frac{11}{2}} \\
& +1566.86 t^{\frac{13}{2}}+162.772 t^{\frac{15}{2}} \\
& +5.336 t^{\frac{17}{2}}+3.639 \times 10^{-2} t^{\frac{19}{2}}-1.081 \times 10^{-3} t^{10}-0.564 t^{9}-34.580 t^{8} \\
& -576.23 t^{7}-3313.03 t^{6} \\
& -7096.13 t^{5}-5595.89 t^{4}-1482.34 t^{3}-107.607 t^{2}-1.39185 t \\
& +0.0607976 \sqrt{t}+0.15 \\
& Z_{20, \frac{1}{2}}(t) \\
& =-13.434 t^{\frac{3}{2}}-429.968 t^{\frac{5}{2}}-3142.94 t^{\frac{7}{2}}-7009.8 t^{\frac{9}{2}}-5513.63 t^{\frac{11}{2}}-1613.32 t^{\frac{13}{2}} \\
& -170.966 t^{\frac{15}{2}} \\
& -5.708 t^{\frac{17}{2}}-3.961 \times 10^{-2} t^{\frac{19}{2}}+1.187 \times 10^{-3} t^{10}+0.608 t^{9}+36.657 t^{8} \\
& +599.43 t^{7}+3373.83 t^{6} \\
& +7045.37 t^{5}+5381.88 t^{4}+1367.07 t^{3}+93.854 t^{2}+1.301 t \\
& -2.709 \times 10^{-2} \sqrt{t}+0.25
\end{aligned}
$$




\section{Conclusions}

This paper adopted a fully fractional-order Legendre collocation method to study a fractional-order version of the differential system of GBM-IS. The powerful numerical scheme gave way to a number of impressive numerical results that prove high efficiency of the algorithm. The results of the algorithm pave way to conduct further additional research in this field to display additional results in the future. Also, we provided numerical simulations exhibiting dynamical behavior and stability around equilibria of the system, and on the basis of simulations and comparison with the experimental data, it is concluded that the fractional-order version of the differential system of GBM-IS is a better representative of the system than its integer-order form. In fact, our approach has three advantages: (i) lower computational cost, (ii) ease of implementation, and (iii) exponential accuracy.

Acknowledgements

Not applicable.

\section{Funding}

This Project was funded by the Deanship of Scientific Research (DSR) at King Abdulaziz University, Jeddah, under grant no. G:253-130-1441. The authors, therefore, acknowledge with thanks DSR for technical and financial support.

Availability of data and materials

Not applicable.

Competing interests

The authors declare that they have no competing interests.

Authors' contributions

All authors prepared, applied the numerical method, made the algorithm, read and approved the final manuscript.

\section{Author details}

${ }^{1}$ Department of Mathematics, Faculty of Science, King Abdulaziz University, Jeddah, Saudi Arabia. ${ }^{2}$ Department of Mathematics, Faculty of Science, Beni-Suef University, Beni-Suef, Egypt. ${ }^{3}$ Department of Mathematics and Statistics, College of Science, Imam Mohammad Ibn Saud Islamic University (IMSIU), Riyadh, Saudi Arabia.

\section{Publisher's Note}

Springer Nature remains neutral with regard to jurisdictional claims in published maps and institutional affiliations.

Received: 30 July 2020 Accepted: 16 September 2020 Published online: 22 September 2020

\section{References}

1. El-Gohary, A.: Chaos and optimal control of cancer selfremission and tumor system steady states. Chaos Solitons Fractals 37, 1305-1316 (2008)

2. Holland, E.C.: Glioblastoma multiforme: the terminator. Proc. Natl. Acad. Sci. 97, 6242-6244 (2000)

3. Yung, Y.A., Shapiro, J.R., Shapiro, W.R.: Heterogeneous chemosensitivities of subpopulations of human glioma cells in culture. Cancer Res. 42, 992-998 (1982)

4. Paulus, W., Peiffer, J.: Intratumoral histologic heterogeneity of gliomas, a quantitative study. Cancer $64,442-447$ (1989)

5. Berkman, R.A., et al.: Clonal composition of glioblastoma multiforme. J. Neurosurg. 77, 432-437 (1992)

6. Hilfer, R.: Applications of Fractional Calculus in Physics. World Scientific, Singapore (2000)

7. El-Gohary, A.: The chaos and optimal control of cancer model with complete unkown parameters. Chaos Solitons Fractals 42, 2865-2874 (2009)

8. Mukhopadhyay, B., Bhattacharyya, R.: Temporal and spatiotemporal variations in a mathematical model of macrophage-tumor interaction. Nonlinear Anal. Hybrid Syst. 2, 819-831 (2008)

9. Rihan, F.A.: Numerical modeling of fractional-order biological systems. Abstr. Appl. Anal. 2013, Article ID 816803 (2013)

10. Ionescu, C., Lopes, A., Copot, D., Tenreiro Machado, J.A., Bates, J.H.T.: The role of fractional calculus in modeling biological phenomena: a review. Commun. Nonlinear Sci. Numer. Simul. 51, 141-159 (2017)

11. Frunzo, L., Garra, R., Giusti, A., Luongo, V.: Modeling biological systems with an improved fractional Gompertz law. Commun. Nonlinear Sci. Numer. Simul. 74, 260-267 (2019)

12. Jin, N., Sun, S.: On a coupled system of fractional compartmental models for a biological system. Adv. Differ. Equ. 2017(1), $146(2017)$

13. Atangana, A., Alabaraoye, E.: Solving a system of fractional partial differential equations arising in the model of HIV infection of CD4+ cells and attractor one-dimensional Keller-Segel equations. Adv. Differ. Equ. (2013). https://doi.org/10.1186/1687-1847-2013-94

14. Magin, R.L.: Fractional Calculus in Bioengineering. Begell House, Redding (2006) 
15. Giona, M., Roman, H.E.: Fractional diffusion equation for transport phenomena in random media. Physica A 185 87-97 (1992)

16. Srivastava, M.H., Ahmad, H., Ahmad, I., Thounthong, P., Khan, N.M.: Numerical simulation of three-dimensional fractional-order convection-diffusion PDEs by a local meshless method. Therm. Sci., 210 (2020)

17. Abo-Dahab, S.M., Abouelregal, A.E., Ahmad, H.. Fractional heat conduction model with phase lags for a half-space with thermal conductivity and temperature dependent. Math. Methods Appl. Sci. (2020). https://doi.org/10.1002/mma.6614

18. Kirchner, J.W., Feng, X., Neal, C.: Fractal stream chemistry and its implications for containant transport in catchments. Nature 403, 524-526 (2000)

19. Podlubny, I.: Fractional differential equations. In: Mathematics in Science and Engineering. Academic Press, San Diego (1999)

20. Meerschaert, M.M., Tadjeran, C.: Finite difference approximations for two-sided space-fractional partial differential equations. Appl. Numer. Math. 56(1), 80-90 (2006)

21. Doha, E.H., Bhrawy, A.H., Ezz-Eldien, S.S.: Efficient Chebyshev spectral methods for solving multi-term fractional orders differential equations. Appl. Math. Model. 35, 5662-5672 (2011)

22. Li, C., Zeng, F:. Finite difference methods for fractional differential equations. Int. J. Bifurc. Chaos 22(4), 1230014 (2012)

23. Kumar, S., Kumar, R., Agarwal, R., Ravi, P., et al.: A study of fractional Lotka-Volterra population model using Haar wavelet and Adams-Bashforth-Moulton methods. Math. Methods Appl. Sci. 43(8), 5564-5578 (2020)

24. Abdelkawy, M.A., Zaky, M.A., Bhrawy, A.H., Baleanu, D.: Numerical simulation of time variable fractional order mobile-immobile advection-dispersion model. Rom. Rep. Phys. 67(3), 773-791 (2015)

25. Doha, E.H.: On the construction of recurrence relations for the expansion and connection coefficients in series of Jacobi polynomials. J. Phys. A, Math. Gen. 37, 657-675 (2004)

26. Bhrawy, A.H., Ahmed, E.A., Baleanu, D.: An efficient collocation technique for solving generalized Fokker-Planck type equations with variable coefficients. Proc. Rom. Acad., Ser. A: Math. Phys. Tech. Sci. Inf. Sci. 15, 322-330 (2014)

27. Bhrawy, A.H.: A Jacobi-Gauss-Lobatto collocation method for solving generalized Fitzhugh-Nagumo equation with time-dependent coefficients. Appl. Math. Comput. 222, 255-264 (2013)

28. Bhrawy, A.H., Zaky, M.A.: Numerical simulation for two dimensional variable-order fractional nonlinear cable equation. Nonlinear Dyn. 80(1-2), 101-116 (2015)

29. Zaky, M.A.: An accurate spectral collocation method for nonlinear systems of fractional differential equations and related integral equations with nonsmooth solutions. Appl. Numer. Math. 154, 205-222 (2020)

30. Abdelkawy, M.A., Lopes, A.M., Zaky, M.: Shifted fractional Jacobi spectral algorithm for solving distributed order time-fractional reaction-diffusion equations. Comput. Appl. Math. 38(2), 81 (2019)

31. Bhrawy, A.H., Doha, E.H., Baleanu, D., Ezz-Eldien, S.S.: A spectral tau algorithm based on Jacobi operational matrix for numerical solution of time fractional diffusion-wave equations. J. Comput. Phys. 293, 142-156 (2015)

32. Hendy, A.S., Zaky, M.A.: Global consistency analysis of L1-Galerkin spectral schemes for coupled nonlinear space-time fractional Schrödinger equations. Appl. Numer. Math. 156, 276-302 (2020)

33. Zayernouri, M., Ainsworth, M., Karniadakis, G.E.: A unified Petrov-Galerkin spectral method for fractional PDEs. Comput. Methods Appl. Mech. Eng. 283, 1545-1569 (2015)

34. Bozkurt, F: Stability analysis of a fractional-order differential equation system of a GBM-IS interaction depending on the density. Appl. Math. Inf. Sci. 8(3), 1021-1028 (2014)

35. Birkhead, B.G., et al.: A mathematical model of the development of drug resistance to cancer chemotherapy. Eur. J. Cancer Clin. Oncol. 23, 1421-1427 (1987)

36. Mukhopadhyay, B., Bhattacharyya, R.: Temporal and spatiotemporal variations in a mathematical model of macrophage-tumor interaction. Nonlinear Anal. Hybrid Syst. 2, 819-831 (2008)

37. Syam, M., Al-Refai, M.: Solving fractional diffusion equation via the collocation method based on fractional Legendre functions. J. Comput. Methods Phys. 2014, Article ID 381074 (2014)

38. Abdelkawy, M.: A collocation method based on Jacobi and fractional order Jacobi basis functions for multi-dimensional distributed-order diffusion equations. Int. J. Nonlinear Sci. Numer. Simul. 19(7-8), 781-792 (2018)

39. Abdelkawy, M., Lopes, A.M., Babatin, M.M.: Shifted fractional Jacobi collocation method for solving fractional functional differential equations of variable order. Chaos Solitons Fractals 134, 109721 (2020)

40. Zaky, M.A., Doha, E.H., Tenreiro Machado, J.A.: A spectral framework for fractional variational problems based on fractional Jacobi functions. Appl. Numer. Math. 132, 51-72 (2018)

41. Odibat, Z.M., Shawagfeh, N.T.: Generalized Taylor's formula. Appl. Math. Comput. 186, 286-293 (2007)

\section{Submit your manuscript to a SpringerOpen ${ }^{\circ}$ journal and benefit from:}

- Convenient online submission

- Rigorous peer review

- Open access: articles freely available online

- High visibility within the field

- Retaining the copyright to your article

Submit your next manuscript at $\gg$ springeropen.com 